\title{
In Vitro Activity of LY127935
}

\author{
ROBERT J. FASS \\ Division of Infectious Diseases, Department of Medicine, The Ohio State University College of Medicine, \\ Columbus, Ohio 43210
}

Received for publication 26 July 1979

\begin{abstract}
LY127935 is a unique new $\beta$-lactam antibiotic. Its activity against 536 clinical isolates was studied by using microdilution methods of susceptibility testing and compared with the activities of cefamandole, cefoxitin, and cephalothin. The lowest concentrations required to inhibit at least $90 \%$ of strains tested (MIC ${ }_{90}$ s) of LY127935 for Staphylococcus aureus, Streptococcus pyogenes, Streptococcus agalactiae, and Streptococcus pneumoniae ranged from 2 to $8 \mu \mathrm{g} / \mathrm{ml}$. The $\mathrm{MIC}_{90} \mathrm{~S}$ for other staphylococci and streptococci were higher. The MIC ${ }_{90}$ s for Enterobacteriaceae and Pseudomonas species ranged from 0.12 to $8 \mu \mathrm{g} / \mathrm{ml}$ and 8 to $>32 \mu \mathrm{g} /$ $\mathrm{ml}$, respectively. The $\mathrm{MIC}_{90}$ for anaerobes ranged from 2 to $>32 \mu \mathrm{g} / \mathrm{ml}$. As determined by $\mathrm{MIC}_{90}$, LY127935 was consistently the least active antibiotic against facultatively anaerobic gram-positive cocci and the most active against aerobic and facultatively anaerobic gram-negative bacilli. Its position with respect to activity against anaerobes varied from being the most active against Bacteroides fragilis and Clostridium perfringens to the least active against anaerobic cocci. In a population of multidrug-resistant isolates, concentrations of $8 \mu \mathrm{g}$ or less of LY127935 per ml inhibited $82 \%$ of Enterobacteriaceae; concentrations of $32 \mu \mathrm{g}$ or less per $\mathrm{ml}$ inhibited $100 \%$ of Enterobacteriaceae and $40 \%$ of $P$. aeruginosa. Increasing the inoculum size by 100 -fold did not increase the minimal inhibitory concentrations of LY127935 or cefoxitin but did increase minimal inhibitory concentrations of cefamandole and cephalothin for some Enterobacteriaceae. All four drugs were bactericidal; minimal bactericidal concentrations were the same or one concentration higher than minimal inhibitory concentrations for 91 to $96 \%$ of strains tested. The broad spectrum and marked in vitro activity of LY127935 make it a promising new antibiotic.
\end{abstract}

LY127935 (compound 6059-S) is a new semisynthetic $\beta$-lactam antibacterial agent which is highly active against a broad spectrum of microorganisms. The replacement of sulfur with oxygen in the six-membered ring of the conventional cephem nucleus makes it unique among currently available $\beta$-lactam antibiotics (Lilly Research Laboratories, unpublished data).

This paper reports the in vitro susceptibilities of a broad spectrum of clinical isolates to LY127935, cefamandole, cefoxitin, and cephalothin.

\section{MATERIALS AND METHODS}

Organisms. The organisms studied included 498 randomly selected and 38 multidrug-resistant recent clinical isolates from patients hospitalized in The Ohio State University Hospitals, Columbus, Ohio. The randomly selected isolates included 86 strains of facultatively anaerobic gram-positive cocci (see Table 1), 271 strains of aerobic and facultatively anaerobic gramnegative bacilli (see Table 2), and 141 strains of anaerobes (see Table 4). Of the 357 aerobic and facultatively anaerobic strains, $60 \%$ were blood isolates; the remainder were isolated from a variety of sources. The anaerobic bacteria were isolated primarily from wounds and abscesses. The multidrug-resistant isolates (see Table 3) included strains of Enterobacteriaceae and Pseudomonas aeruginosa which were resistant to $64 \mu \mathrm{g}$ of ampicillin, $128 \mu \mathrm{g}$ of carbenicillin, 4 $\mu \mathrm{g}$ of gentamicin, and $8 \mu \mathrm{g}$ of tetracycline per $\mathrm{ml}$.

Antibiotics. The antibiotics tested included LY127935, cefamandole, and cephalothin, which were obtained from Lilly Research Laboratories, Indianapolis, Ind., and cefoxitin, which was obtained from Merck, Sharp \& Dohme, West Point, Pa. Laboratory standard powders were diluted as recommended by the manufacturers to stock concentrations of $1,000 \mu \mathrm{g} /$ $\mathrm{ml}$ and used immediately or frozen at $-20^{\circ} \mathrm{C}$, for up to 1 month, until used.

Susceptibility tests. Minimal inhibitory concentrations (MICs) of the four study antibiotics were determined simultaneously and in duplicate for each isolate by microdilution methods. For aerobic and facultatively anaerobic organisms, antibiotics were diluted in broth and dispensed into microdilution plates with a Dynatech MIC-2000 (Dynatech Laboratories, Alexandria, Va.) dispensing instrument (3). Antibioticcontaining plates were stored at $-20^{\circ} \mathrm{C}$ until used. For anaerobic organisms, a previously described method 
(1) was used. Antibiotics were diluted in microdilution plates with a Medimixer (Flow Laboratories, Rockville, Md.) automatic diluter on the day of their use. Antibiotic concentrations consisted of $\log _{2}$ dilutions which ranged from 32 to $0.01 \mu \mathrm{g} / \mathrm{ml}$.

For aerobic and facultative gram-positive cocci and anaerobes, MICs were performed in Schaedler broth (BBL Microbiology Systems, Cockeysville, Md.); with Bacteroides melaninogenicus, Fusobacterium, and anaerobic cocci it was supplemented to contain $1 \%$ heat-inactivated $\left(56^{\circ} \mathrm{C}\right.$ for $\left.30 \mathrm{~min}\right)$ horse serum. For aerobic and facultative gram-negative bacilli, MICs were performed in Mueller-Hinton broth (Difco Laboratories, Detroit, Mich.) supplemented with $\mathrm{CaCl}_{2}$ and $\mathrm{MgCl}_{2}$ to contain $5 \mathrm{mg}$ of calcium and $2.5 \mathrm{mg}$ of magnesium per $\mathrm{dl}$, respectively.

Antibiotic-containing microdilution plates were inoculated with MIC-2000 disposable inoculators (Dynatech) so that the final inoculum was approximately $10^{5}$ to $5 \times 10^{5}$ colony-forming units per ml. Inoculated plates were incubated at $37^{\circ} \mathrm{C}$ in either a room air incubator (aerobes and facultatives other than Streptococcus pyogenes, Streptococcus pneumoniae, and
Streptococcus viridans) a $5 \% \mathrm{CO}_{2}$ incubator (S. pyogenes, $S$. pneumoniae, and $S$. viridans), or an anaerobic glove-box containing $85 \% \mathrm{~N}_{2}, 10 \% \mathrm{H}_{2}$, and $5 \% \mathrm{CO}_{2}$ (anaerobes). After 16 to $20 \mathrm{~h}$ (aerobes and facultatives) or $48 \mathrm{~h}$ (anaerobes) of incubation, plates were read with the aid of a magnifying mirror. The lowest concentrations of antibiotic which inhibited all visually apparent growth was considered the MIC. A control strain, Escherichia coli ATCC 25922 or Bacteroides fragilis ID 00170, was included with each group of organisms tested. For each antibiotic-organism combination, the $\mathrm{MIC}_{50}$ was the lowest concentration of antibiotic which inhibited at least $50 \%$ of the strains tested, and the $\mathrm{MIC}_{90}$ was the lowest concentration which inhibited at least 90 of strains tested.

To determine the effects of varying inoculum size on MICs, three strains each of nine organisms (see Table 5) were studied. MICs of the four study antibiotics were simultaneously determined with a standard inoculum, and inocula containing 100 -fold higher and 100-fold lower colony-forming units per milliliter.

To determine minimal bactericidal concentrations (MBCs), wells from 116 MIC plates (10 strains of

TABLE 1. Comparative in vitro activity of LY127935, cefamandole, cefoxitin, and cephalothin against staphylococci and streptococci

\begin{tabular}{|c|c|c|c|c|}
\hline $\begin{array}{c}\text { Organism (no. of } \\
\text { strains) }\end{array}$ & Antibiotic & MIC range $(\mu \mathrm{g} / \mathrm{ml})$ & $\mathrm{MIC}_{\mathrm{n}}\left(\mu_{\mathrm{g}} / \mathrm{ml}\right)$ & $\operatorname{MIC}_{s \mathrm{n}}(\mu \mathrm{g} / \mathrm{ml})$ \\
\hline Staphylococcus aureus (24) & $\begin{array}{l}\text { LY127935 } \\
\text { Cefamandole } \\
\text { Cefoxitin } \\
\text { Cephalothin }\end{array}$ & $\begin{array}{c}4-8 \\
0.12-1 \\
2-4 \\
0.12-0.25\end{array}$ & $\begin{array}{l}8 \\
0.5 \\
2 \\
0.12\end{array}$ & $\begin{array}{l}8 \\
0.5 \\
4 \\
0.25\end{array}$ \\
\hline Staphylococcus epidermidis (10) & $\begin{array}{l}\text { LY127935 } \\
\text { Cefamandole } \\
\text { Cefoxitin } \\
\text { Cephalothin }\end{array}$ & $\begin{array}{c}16->32 \\
0.25-1 \\
2-32 \\
0.12->32\end{array}$ & $\begin{array}{l}32 \\
0.5 \\
8 \\
0.25\end{array}$ & $\begin{array}{c}32 \\
0.5 \\
16 \\
0.5\end{array}$ \\
\hline Streptococcus pyogenes (10) & $\begin{array}{l}\text { LY127935 } \\
\text { Cefamandole } \\
\text { Cefoxitin } \\
\text { Cephalothin }\end{array}$ & \begin{tabular}{l}
\multicolumn{1}{c}{$1-2$} \\
$0.03-0.06$ \\
$0.12-0.5$ \\
$0.06-0.12$
\end{tabular} & $\begin{array}{l}1 \\
0.06 \\
0.5 \\
0.12\end{array}$ & $\begin{array}{l}2 \\
0.06 \\
0.5 \\
0.12\end{array}$ \\
\hline Streptococcus agalactiae (10) & $\begin{array}{l}\text { LY127935 } \\
\text { Cefamandole } \\
\text { Cefoxitin } \\
\text { Cephalothin }\end{array}$ & $\begin{array}{c}4-8 \\
0.03-0.12 \\
1-4 \\
0.06-0.12\end{array}$ & $\begin{array}{l}4 \\
0.06 \\
2 \\
0.12\end{array}$ & $\begin{array}{l}8 \\
0.06 \\
4 \\
0.12\end{array}$ \\
\hline Streptococcus pneumoniae (11) & $\begin{array}{l}\text { LY127935 } \\
\text { Cefamandole } \\
\text { Cefoxitin } \\
\text { Cephalothin }\end{array}$ & $\begin{aligned} & 0.03-4 \\
\leq & 0.01-0.12 \\
\leq & 0.01-2 \\
\leq & 0.01-0.12\end{aligned}$ & $\begin{array}{r}0.5 \\
\leq 0.01 \\
0.12 \\
\leq 0.01\end{array}$ & $\begin{array}{l}2 \\
0.06 \\
0.5 \\
0.06\end{array}$ \\
\hline Streptococcus viridans (10) & $\begin{array}{l}\text { LY127935 } \\
\text { Cefamandole } \\
\text { Cefoxitin } \\
\text { Cephalothin }\end{array}$ & $\begin{array}{l}\quad 1->32 \\
0.06-1 \\
0.06-8 \\
0.06-0.12\end{array}$ & $\begin{array}{l}8 \\
0.12 \\
2 \\
0.12\end{array}$ & $\begin{array}{l}32 \\
0.12 \\
8 \\
0.12\end{array}$ \\
\hline Streptococcus faecalis (11) & $\begin{array}{l}\text { LY127935 } \\
\text { Cefamandole } \\
\text { Cefoxitin } \\
\text { Cephalothin }\end{array}$ & $\begin{array}{c}>32 \\
16->32 \\
>32 \\
8-32\end{array}$ & $\begin{array}{r}>32 \\
32 \\
>32 \\
16\end{array}$ & $\begin{array}{r}>32 \\
32 \\
>32 \\
32\end{array}$ \\
\hline
\end{tabular}


TABLE 2. Comparative in vitro activity of LY127935, cefamandole, cefoxitin, and cephalothin against Enterobacteriaceae and Pseudomonas

\begin{tabular}{|c|c|c|c|c|}
\hline Organism (no. of strains) & Antibiotic & MIC range ( $\mu \mathrm{g} / \mathrm{ml})$ & $\mathrm{MIC}_{50}(\mu \mathrm{g} / \mathrm{ml})$ & $\mathrm{MIC}_{90}(\mu \mathrm{g} / \mathrm{ml})$ \\
\hline Escherichia coli (25) & $\begin{array}{l}\text { LY127935 } \\
\text { Cefamandole } \\
\text { Cefoxitin } \\
\text { Cephalothin }\end{array}$ & $\begin{array}{c}0.03-0.25 \\
0.06-4 \\
0.5-2 \\
0.5-8\end{array}$ & $\begin{array}{l}0.12 \\
0.25 \\
1 \\
4\end{array}$ & $\begin{array}{l}0.12 \\
1 \\
2 \\
8\end{array}$ \\
\hline Klebsiella pneumoniae (25) & $\begin{array}{l}\text { LY127935 } \\
\text { Cefamandole } \\
\text { Cefoxitin } \\
\text { Cephalothin }\end{array}$ & $\begin{array}{c}0.06-0.5 \\
0.12-8 \\
0.5-4 \\
1-32\end{array}$ & $\begin{array}{l}0.12 \\
0.5 \\
2 \\
2\end{array}$ & $\begin{array}{l}0.25 \\
1 \\
2 \\
4\end{array}$ \\
\hline Enterobacter aerogenes (20) & $\begin{array}{l}\text { LY127935 } \\
\text { Cefamandole } \\
\text { Cefoxitin } \\
\text { Cephalothin }\end{array}$ & $\begin{array}{l}0.06-8 \\
0.25->32 \\
8->32 \\
>32\end{array}$ & $\begin{aligned} & 0.25 \\
& 1 \\
> & 32 \\
> & 32\end{aligned}$ & $\begin{array}{r}\quad 4 \\
>32 \\
>32 \\
>32\end{array}$ \\
\hline Enterobacter cloacae (24) & $\begin{array}{l}\text { LY127935 } \\
\text { Cefamandole } \\
\text { Cefoxitin } \\
\text { Cephalothin }\end{array}$ & $\begin{array}{c}0.06-32 \\
0.5->32 \\
32->32 \\
32->32\end{array}$ & $\begin{aligned} & 0.12 \\
& 2 \\
> & 32 \\
> & 32\end{aligned}$ & $\begin{array}{r}8 \\
>32 \\
>32 \\
>32\end{array}$ \\
\hline Serratia marcescens (25) & $\begin{array}{l}\text { LY127935 } \\
\text { Cefamandole } \\
\text { Cefoxitin } \\
\text { Cephalothin }\end{array}$ & $\begin{array}{c}0.25-16 \\
8->32 \\
4->32 \\
>32\end{array}$ & $\begin{array}{r}0.5 \\
>32 \\
8 \\
>32\end{array}$ & $\begin{array}{r}2 \\
>32 \\
>32 \\
>32\end{array}$ \\
\hline Citrobacter diversus (15) & $\begin{array}{l}\text { LY127935 } \\
\text { Cefamandole } \\
\text { Cefoxitin } \\
\text { Cephalothin }\end{array}$ & $\begin{array}{c}0.12-1 \\
0.12->32 \\
0.5-16 \\
1->32\end{array}$ & $\begin{array}{l}0.12 \\
8 \\
2 \\
2\end{array}$ & $\begin{array}{r}0.5 \\
32 \\
8 \\
>32\end{array}$ \\
\hline Citrobacter freundii (16) & $\begin{array}{l}\text { LY127935 } \\
\text { Cefamandole } \\
\text { Cefoxitin } \\
\text { Cephalothin }\end{array}$ & $\begin{array}{l}0.06-2 \\
0.25->32 \\
32->32 \\
32->32\end{array}$ & $\begin{aligned} & 0.12 \\
& 0.5 \\
> & 32 \\
> & 32\end{aligned}$ & $\begin{array}{r}2 \\
>32 \\
>32 \\
>32\end{array}$ \\
\hline Proteus mirabilis (22) & $\begin{array}{l}\text { LY127935 } \\
\text { Cefamandole } \\
\text { Cefoxitin } \\
\text { Cephalothin }\end{array}$ & $\begin{array}{c}0.06-0.25 \\
0.12-0.5 \\
1-2 \\
1-2\end{array}$ & $\begin{array}{l}0.12 \\
0.25 \\
2 \\
2\end{array}$ & $\begin{array}{l}0.25 \\
0.5 \\
2 \\
2\end{array}$ \\
\hline Proteus vulgaris (16) & $\begin{array}{l}\text { LY127935 } \\
\text { Cefamandole } \\
\text { Cefoxitin } \\
\text { Cephalothin }\end{array}$ & $\begin{array}{c}0.12-1 \\
1->32 \\
1-8 \\
4->32\end{array}$ & $\begin{array}{c}0.25 \\
>32 \\
2 \\
>32\end{array}$ & $\begin{array}{r}0.5 \\
>32 \\
8 \\
>32\end{array}$ \\
\hline Proteus rettgeri (10) & $\begin{array}{l}\text { LY127935 } \\
\text { Cefamandole } \\
\text { Cefoxitin } \\
\text { Cephalothin }\end{array}$ & $\begin{array}{r}0.06-4 \\
0.06->32 \\
0.5->32 \\
1->32\end{array}$ & $\begin{aligned} & 0.12 \\
& 0.5 \\
2 & \\
> & 32\end{aligned}$ & $\begin{aligned} & 0.25 \\
& 16 \\
> & 32 \\
> & 32\end{aligned}$ \\
\hline Providencia stuartii (18) & $\begin{array}{l}\text { LY127935 } \\
\text { Cefamandole } \\
\text { Cefoxitin } \\
\text { Cephalothin }\end{array}$ & $\begin{array}{l}0.06-1 \\
0.12-32 \\
0.5->32 \\
1->32\end{array}$ & $\begin{aligned} & 0.12 \\
& 0.5 \\
2 & \\
> & 32\end{aligned}$ & $\begin{aligned} & 0.5 \\
& 8 \\
& 16 \\
&> 32\end{aligned}$ \\
\hline Morganella morganii (15) & $\begin{array}{l}\text { LY127935 } \\
\text { Cefamandole } \\
\text { Cefoxitin } \\
\text { Cephalothin }\end{array}$ & $\begin{array}{c}0.12-0.5 \\
2->32 \\
4->32 \\
>32\end{array}$ & $\begin{aligned} & 0.25 \\
& 8 \\
& 8 \\
&> 32\end{aligned}$ & $\begin{array}{c}\quad 0.5 \\
32 \\
32 \\
>32\end{array}$ \\
\hline
\end{tabular}


TABLE 2-Continued

\begin{tabular}{|c|c|c|c|c|}
\hline Organism (no. of strains) & Antibiotic & MIC range $(\mu \mathrm{g} / \mathrm{ml})$ & $\operatorname{MIC}_{[n}(\mu \mathrm{g} / \mathrm{ml})$ & $\mathrm{MIC}_{20}(\mu \mathrm{g} / \mathrm{ml})$ \\
\hline Pseudomonas aeruginosa (20) & $\begin{array}{l}\text { LY127935 } \\
\text { Cefamandole } \\
\text { Cefoxitin } \\
\text { Cephalothin }\end{array}$ & $\begin{aligned} & 4->32 \\
> & 32 \\
> & 32 \\
> & 32\end{aligned}$ & $\begin{array}{r}16 \\
>32 \\
>32 \\
>32\end{array}$ & $\begin{array}{r}32 \\
>32 \\
>32 \\
>32\end{array}$ \\
\hline Pseudomonas maltophilia (10) & $\begin{array}{l}\text { LY127935 } \\
\text { Cefamandole } \\
\text { Cefoxitin } \\
\text { Cephalothin }\end{array}$ & $\begin{aligned} & 4-32 \\
> & 32 \\
> & 32 \\
> & 32\end{aligned}$ & $\begin{array}{r}8 \\
>32 \\
>32 \\
>32\end{array}$ & $\begin{array}{r}8 \\
>32 \\
>32 \\
>32\end{array}$ \\
\hline Pseudomonas cepacia (10) & $\begin{array}{l}\text { LY127935 } \\
\text { Cefamandole } \\
\text { Cefoxitin } \\
\text { Cephalothin }\end{array}$ & $\begin{array}{l}32->32 \\
32->32 \\
16->32 \\
32->32\end{array}$ & $\begin{array}{l}>32 \\
>32 \\
>32 \\
>32\end{array}$ & $\begin{array}{l}>32 \\
>32 \\
>32 \\
>32\end{array}$ \\
\hline
\end{tabular}

Staphylococcus aureus, 6 strains of Staphylococcus epidermidis, 75 strains of Enterobacteriaceae, 12 strains of $P$. aeruginosa, and 13 strains of $B$. fragilis) were subcultured to microdilution plates containing sterile broth with disposable inoculators. The absence of growth after incubation of the subculture from a given well of an MIC plate indicated a reduction in the original number of colony-forming units of $99.9 \%$ or greater in that well. MBCs were the lowest concentrations of antibiotic which yielded no growth in subculture after overnight incubation.

\section{RESULTS}

The comparative in vitro activities of the four study antibiotics against staphylococci and streptococci are shown in Table 1. Cephalothin and cefamandole were most active, cefoxitin was intermediate in activity, and LY127935 was the least active drug studied. Concentrations of $8 \mu \mathrm{g}$ of LY127935 per $\mathrm{ml}$ inhibited $100 \%$ of strains of $S$. aureus, $S$. pyogenes, $S$. agalactiae, and $S$. pneumoniae but not $S$. epidermidis, $S$. viridans, or S. faecalis.

The comparative in vitro activities of the four study antibiotics against randomly selected $\boldsymbol{E n}$. terobacteriaceae and three species of Pseudom. onas are shown in Table 2. LY127935 was the most active drug against all organisms tested. Concentrations of $8 \mu \mathrm{g} / \mathrm{ml}$ inhibited $98.7 \%$ of Enterobacteriaceae. Concentrations of $32 \mu \mathrm{g} / \mathrm{ml}$ inhibited $99.6 \%$ of Enterobacteriaceae and Pseudomonas other than P. cepacia.

The in vitro susceptibilities of multidrug-resistant Enterobacteriaceae and $P$. aeruginosa are shown in Table 3 . In addition to resistance to ampicillin, carbenicillin, gentamicin, and tetracycline, most strains were also resistant to cephalothin, cefamandole, cefoxitin, tobramycin, and chloramphenicol, but $89 \%$ of the Enterobac- teriaceae and 6 of the 10 strains of $P$. aeruginosa were inhibited by $16 \mu \mathrm{g}$ of amikacin per $\mathrm{ml}$. Concentrations of $8 \mu \mathrm{g}$ of LY127935 per $\mathrm{ml}$ inhibited $82 \%$ of the Enterobacteriaceae. Concentrations of $32 \mu \mathrm{g} / \mathrm{ml}$ inhibited $100 \%$ of Enterobacteriaceae and 4 of 10 strains of $P$. aeruginosa.

The comparative in vitro activities of the four study antibiotics against anaerobes are shown in Table 4. LY127935 was the most active drug tested against Bacteroides and Clostridium perfringens; $100 \%$ of strains of $B$. fragilis and $C$. perfringens tested were inhibited by $2 \mu \mathrm{g} / \mathrm{ml}$. Although it was usually less active than the other drugs against other anaerobes, particularly the anaerobic cocci, $87 \%$ of all anaerobes tested were inhibited by $8 \mu \mathrm{g} / \mathrm{ml}$ and $96 \%$ were inhibited by $32 \mu \mathrm{g} / \mathrm{ml}$.

Lowering the inoculum size 100 -fold had no effect on the MICs of the four study antibiotics. The effects of increasing the inoculum size 100fold to approximately $10^{7}$ to $5 \times 10^{7}$ colonyforming units per $\mathrm{ml}$ are shown in Table 5. With cephalothin and cefamandole, increases in MICs of more than two concentrations were observed with some susceptible organisms. Such increases were not observed with LY127935 or cefoxitin.

The four antibiotics studied were bactericidal against all 11 species of organisms studied. MBCs were the same or $1 \log _{2}$ dilution higher for 91 to $96 \%$ of the strains tested. MBCs were within $2 \log _{2}$ dilutions of MICs for $99 \%$ of strains with all four antibiotics.

\section{DISCUSSION}

LY127935 had a broad spectrum of activity against a variety of clinical isolates. Although it was less active than cefamandole, cefoxitin, and 
TABLE 3. In vitro susceptibilities of multidrug-resistant "Enterobacteriaceae and P. aeruginosa

\begin{tabular}{|c|c|c|c|c|c|c|c|}
\hline \multirow[b]{2}{*}{ Organism } & \multicolumn{7}{|c|}{ MICs $(\mu \mathrm{g} / \mathrm{ml})$} \\
\hline & LY127935 & Cefamandole & Cefoxitin & Cephalothin & Tobramycin & Amikacin & $\begin{array}{l}\text { Chloram- } \\
\text { phenicol }\end{array}$ \\
\hline \multirow[t]{2}{*}{ E. coli } & 0.12 & 4 & 1 & 16 & $>16$ & 16 & 8 \\
\hline & 0.5 & 2 & 2 & 8 & $>16$ & $>32$ & 4 \\
\hline \multirow[t]{5}{*}{ K. pneumoniae } & 0.25 & 4 & 8 & 16 & $>16$ & 16 & $>32$ \\
\hline & 0.25 & 32 & 1 & $>32$ & $>16$ & 1 & 2 \\
\hline & 2 & 16 & 32 & $>32$ & $>16$ & 2 & $>32$ \\
\hline & 4 & 16 & 32 & $>32$ & $>16$ & 2. & $>32$ \\
\hline & 32 & $>32$ & $>32$ & $>32$ & 8 & 32 & $>32$ \\
\hline \multirow[t]{4}{*}{ E. aerogenes } & 8 & $>32$ & $>32$ & $>32$ & 16 & 1 & $>32$ \\
\hline & 8 & $>32$ & $>32$ & $>32$ & $>16$ & 2 & $>32$ \\
\hline & 8 & $>32$ & $>32$ & $>32$ & $>16$ & 2 & 4 \\
\hline & 16 & $>32$ & $>32$ & $>32$ & $>16$ & 4 & $>32$ \\
\hline \multirow[t]{5}{*}{ E. cloaceae } & 0.25 & $>32$ & $>32$ & $>32$ & $>16$ & 2 & 8 \\
\hline & 0.25 & $>32$ & $>32$ & $>32$ & $>16$ & 2 & $>32$ \\
\hline & 0.25 & $>32$ & $>32$ & $>32$ & $>16$ & 2 & $>32$ \\
\hline & 0.25 & $>32$ & $>32$ & $>32$ & $>16$ & 4 & $>32$ \\
\hline & 0.5 & $>32$ & $>32$ & $>32$ & $>16$ & 2 & $>32$ \\
\hline \multirow[t]{5}{*}{ S. marcescens } & 2 & $>32$ & $>32$ & $>32$ & $>16$ & 4 & 32 \\
\hline & 8 & $>32$ & $>32$ & $>32$ & $>16$ & 4 & 32 \\
\hline & 16 & $>32$ & $>32$ & $>32$ & $>16$ & 4 & 16 \\
\hline & 16 & $>32$ & $>32$ & $>32$ & $>16$ & $>32$ & 32 \\
\hline & 32 & $>32$ & $>32$ & $>32$ & $>16$ & 4 & 16 \\
\hline \multirow[t]{2}{*}{ C. diversus } & 0.5 & 32 & 8 & $>32$ & $>16$ & 8 & 16 \\
\hline & 0.5 & $>32$ & 8 & $>32$ & $>16$ & 2 & 16 \\
\hline \multirow[t]{3}{*}{ P. rettgeri } & 0.12 & 1 & 2 & $>32$ & 16 & 4 & $-{ }^{b}$ \\
\hline & 0.12 & 16 & $>32$ & $>32$ & $>16$ & 2 & - \\
\hline & 0.12 & 16 & $>32$ & $>32$ & $>16$ & 16 & - \\
\hline \multirow[t]{2}{*}{ P. stuartii } & 0.12 & 1 & 1 & $>32$ & 8 & 8 & - \\
\hline & 2 & $>32$ & $>32$ & $>32$ & 8 & 2 & 16 \\
\hline \multirow[t]{10}{*}{$P$. aeruginosa } & 16 & $>32$ & $>32$ & $>32$ & 4 & 32 & $>32$ \\
\hline & 32 & $>32$ & $>32$ & $>32$ & 2 & 16 & $>32$ \\
\hline & 32 & $>32$ & $>32$ & $>32$ & 4 & 16 & $>32$ \\
\hline & 32 & $>32$ & $>32$ & $>32$ & $>16$ & 16 & $>32$ \\
\hline & $>32$ & $>32$ & $>32$ & $>32$ & 4 & 16 & $>32$ \\
\hline & $>32$ & $>32$ & $>32$ & $>32$ & 4 & 32 & $>32$ \\
\hline & $>32$ & $>32$ & $>32$ & $>32$ & $>16$ & 16 & $>32$ \\
\hline & $>32$ & $>32$ & $>32$ & $>32$ & $>16$ & 16 & $>32$ \\
\hline & $>32$ & $>32$ & $>32$ & $>32$ & $>16$ & 32 & $>32$ \\
\hline & $>32$ & $>32$ & $>32$ & $>32$ & 16 & $>32$ & $>32$ \\
\hline
\end{tabular}

" Multidrug-resistant, MICs: ampicillin, $>64 \mu \mathrm{g} / \mathrm{ml}$; carbenicillin, $>128 \mu \mathrm{g} / \mathrm{ml}$; gentamicin, $>4 \mu \mathrm{g} / \mathrm{ml} ;$ and tetracycline, $>8 \mu \mathrm{g} / \mathrm{ml}$.

- Not tested.

cephalothin against staphylococci, streptococci, and some anaerobes, concentrations of $8 \mu \mathrm{g}$ or less per ml inhibited most strains tested excluding $S$. epidermidis, $S$. viridans, and $S$. faecalis. It was the most active drug tested against $E$ nterobacteriaceae, Pseudomonas, B. fragilis, and $C$. perfringens. In a population of multidrug- resistant, gram-negative bacilli, all Enterobacteriaceae and $40 \%$ of strains of $P$. aeruginosa were inhibited by $32 \mu \mathrm{g}$ or less per $\mathrm{ml}$. MICs of LY127935 were not affected by increasing inoculum size, although MICs of cefamandole and cephalothin for some Enterobacteriaceae increased considerably in this study and others 
TABLE 4. Comparative in vitro activity of LY127935, cefamandole, cefoxitin, and cephalothin against anaerobes

\begin{tabular}{|c|c|c|c|c|}
\hline Organism (no. of strains) & Antibiotic & MIC range $(\mu \mathrm{g} / \mathrm{ml})$ & $\operatorname{MIC}_{: n}(\mu \mathrm{g} / \mathrm{ml})$ & $\operatorname{MIC}_{m 0}(\mu \mathrm{g} / \mathrm{ml})$ \\
\hline Bacteroides fragilis (15) & $\begin{array}{l}\text { LY127935 } \\
\text { Cefamandole } \\
\text { Cefoxitin } \\
\text { Cephalothin }\end{array}$ & $\begin{array}{c}0.12-2 \\
16->32 \\
2-8 \\
32->32\end{array}$ & $\begin{array}{r}1 \\
16 \\
4 \\
>32\end{array}$ & $\begin{array}{r}2 \\
32 \\
8 \\
>32\end{array}$ \\
\hline Bacteroides fragilis group ${ }^{a}$ (14) & $\begin{array}{l}\text { LY127935 } \\
\text { Cefamandole } \\
\text { Cefoxitin } \\
\text { Cephalothin }\end{array}$ & $\begin{array}{c}0.5-16 \\
16->32 \\
2-32 \\
16->32\end{array}$ & $\begin{array}{r}4 \\
32 \\
16 \\
>32\end{array}$ & $\begin{array}{r}16 \\
>32 \\
16 \\
>32\end{array}$ \\
\hline Bacteroides melaninogenicus (15) & $\begin{array}{l}\text { LY127935 } \\
\text { Cefamandole } \\
\text { Cefoxitin } \\
\text { Cephalothin }\end{array}$ & $\begin{array}{l}0.03-4 \\
0.03-4 \\
0.06-4 \\
0.03-4\end{array}$ & $\begin{array}{l}0.5 \\
0.25 \\
0.25 \\
0.12\end{array}$ & $\begin{array}{l}2 \\
4 \\
1 \\
2\end{array}$ \\
\hline Fusobacterium (16) & $\begin{array}{l}\text { LY127935 } \\
\text { Cefamandole } \\
\text { Cefoxitin } \\
\text { Cephalothin }\end{array}$ & $\begin{array}{c}0.25-16 \\
\leq 0.01-32 \\
0.06-8 \\
\leq 0.01-32\end{array}$ & $\begin{array}{l}1 \\
0.06 \\
0.12 \\
0.12\end{array}$ & $\begin{array}{l}8 \\
8 \\
8 \\
4\end{array}$ \\
\hline Peptococcus (12) & $\begin{array}{l}\text { LY127935 } \\
\text { Cefamandole } \\
\text { Cefoxitin } \\
\text { Cephalothin }\end{array}$ & $\begin{array}{r}0.12-8 \\
\leq 0.01-2 \\
\leq 0.01-4 \\
\leq 0.01-1\end{array}$ & $\begin{array}{l}1 \\
1 \\
0.12 \\
0.12\end{array}$ & $\begin{array}{l}8 \\
1 \\
0.25 \\
0.5\end{array}$ \\
\hline Peptostreptococcus (13) & $\begin{array}{l}\text { LY127935 } \\
\text { Cefamandole } \\
\text { Cefoxitin } \\
\text { Cephalothin }\end{array}$ & $\begin{array}{c}\quad 0.5->32 \\
0.12-2 \\
0.25-8 \\
\leq 0.01-2\end{array}$ & $\begin{array}{l}4 \\
0.5 \\
1 \\
0.12\end{array}$ & $\begin{array}{r}16 \\
2 \\
8 \\
1\end{array}$ \\
\hline Veillonella (11) & $\begin{array}{l}\text { LY127935 } \\
\text { Cefamandole } \\
\text { Cefoxitin } \\
\text { Cephalothin }\end{array}$ & $\begin{array}{l}0.5-16 \\
0.12-2 \\
0.25-8 \\
0.25-4\end{array}$ & $\begin{array}{l}8 \\
0.5 \\
1 \\
2\end{array}$ & $\begin{array}{r}16 \\
2 \\
4 \\
2\end{array}$ \\
\hline Clostridium perfringens (15) & $\begin{array}{l}\text { LY127935 } \\
\text { Cefamandole } \\
\text { Cefoxitin } \\
\text { Cephalothin }\end{array}$ & $\begin{array}{r}0.25-2 \\
0.5-4 \\
1-2 \\
1-8\end{array}$ & $\begin{array}{l}0.5 \\
2 \\
2 \\
2\end{array}$ & $\begin{array}{l}2 \\
4 \\
2 \\
4\end{array}$ \\
\hline Clostridium sp. (other) (15) & $\begin{array}{l}\text { LY127935 } \\
\text { Cefamandole } \\
\text { Cefoxitin } \\
\text { Cephalothin }\end{array}$ & $\begin{array}{r}2->32 \\
0.25->32 \\
0.25->32 \\
0.25->32\end{array}$ & $\begin{array}{l}4 \\
1 \\
1 \\
1\end{array}$ & $\begin{array}{l}>32 \\
>32 \\
>32 \\
>32\end{array}$ \\
\hline $\begin{array}{l}\text { Gram-positive, nonsporeforming ba- } \\
\text { cilli }^{b}(15)\end{array}$ & $\begin{array}{l}\text { LY127935 } \\
\text { Cefamandole } \\
\text { Cefoxitin } \\
\text { Cephalothin }\end{array}$ & $\begin{array}{c}0.25->32 \\
\leq 0.01-16 \\
0.12->32 \\
\leq 0.01-16\end{array}$ & $\begin{array}{l}1 \\
0.12 \\
0.25 \\
0.5\end{array}$ & $\begin{array}{r}32 \\
2 \\
16 \\
8\end{array}$ \\
\hline
\end{tabular}

\footnotetext{
${ }^{a} B$. fragilis group includes $B$. vulgatus, $B$. thetaiotaomicron, and $B$. distasonis.

${ }^{b}$ Gram-positive nonsporeforming bacilli includes Eubacterium, Bifidobacterium, Lactobacillus, and Propionibacterium.
} 
TABLE 5. Increases in MICs caused by increasing inoculum size 100-fold

\begin{tabular}{|c|c|c|c|c|c|}
\hline \multirow{2}{*}{ Organism } & \multicolumn{5}{|c|}{ Increases in MICs (no. of log.2 dilutions) } \\
\hline & LY127935 & Cefamandole & Cefoxitin & \multicolumn{2}{|c|}{ Cephalothin } \\
\hline S. aureus & $0,1, \quad 1^{\prime \prime}$ & $1, \quad 2, \quad 0$ & 2,0 & 1 & 2, \\
\hline E. coli & $0,0, \quad 1$ & $0, \quad 1$ & $0, \quad 1$ & 4, & $2, \quad \geq 2$ \\
\hline K. pneumoniae & $1,0, \quad 1$ & $6, \geq 5,2$ & $\begin{array}{lll}0, & 0, & 0\end{array}$ & 3 & $\geq 3,3$ \\
\hline$E$. aerogenes & $0,1, \quad 0$ & $1, \quad 2, \quad 1$ & $\mathrm{U}, \geq 2, \mathrm{U}^{b}$ & $\geq 2$, & $\geq 2, \geq 3$ \\
\hline E. cloacae & $0,0,0$ & $\geq 7, \geq 5, \geq 4$ & $\mathrm{U}, \mathbf{U}, \quad \mathbf{U}$ & $\mathrm{U}$ & $\mathrm{U}, \mathrm{U}$ \\
\hline S. marcescens & $0,0,0$ & $\mathrm{U}, \quad \mathrm{U}, \geq 1$ & $0,1, \quad 1$ & $\mathrm{U}$ & $\mathrm{U}, \quad \mathrm{U}$ \\
\hline P. mirabilis & $2,2, \quad 1$ & $1, \quad 1, \quad 0$ & 1,0 & 1 & 0 \\
\hline P. aeruginosa & $1, \geq 1,0$ & $\mathrm{U}, \mathrm{U}, \mathrm{U}$ & $\mathbf{U}, \mathbf{U}, \quad \mathbf{U}$ & $\mathrm{U}$ & $\mathrm{U}, \quad \mathrm{U}$ \\
\hline B. fragilis & $0,0, \quad 0$ & $\geq 2, \geq 1, \geq 1$ & $0,1, \quad 1$ & $\mathrm{U}$ & $\mathrm{U}, \quad \mathrm{U}$ \\
\hline
\end{tabular}

" Each number represents the increase for one of the three strains tested.

${ }^{b} \mathrm{U}$, Undetermined: MIC $>32 \mu \mathrm{g} / \mathrm{ml}$ with an inoculum of $1 \times 10^{5}$ to $5 \times 10^{5}$ colony-forming units per ml.

(2). The antimicrobial activity of LY127935 was bactericidal, as it was for the comparative drugs.

The broad spectrum and marked activity of LY127935, particularly against aerobic, facultative, and anaerobic gram-negative enteric bacilli, make it a promising new antibiotic.

\section{ACKNOWLEDGMENTS}

I thank Valerie L. Helsel for technical assistance.

This work was supported by a grant from Lilly Research Laboratories, Indianapolis, Ind.

\section{LTERATURE CITED}

1. Fass, R. J. 1978. Antimicrobial susceptibility testing of anaerobic bacteria using microdilution techniques. Infect. Dis. Rev. 5:25-42.

2. Findell, C. M., and J. C. Sherris. 1976. Susceptibility of Enterobacter to cefamandole: evidence for a high mutation rate to resistance. Antimicrob. Agents Chemother. 9:970-974.

3. McMaster, P.R.B., E.A. Robertson, F.G. Witebsky, and J.D. MacLowry. 1978. Evaluation of a dispensing instrument (Dynatech MIC-2000) for preparing microtiter antibiotic plates and testing their potency during storage. Antimicrob. Agents Chemother. 13:842-844. 